\title{
SPACES OF MEASURES RELATED TO DEQUANTIZATION
}

\author{
M. Zarichnyi \\ Department of Mechanics and Mathematics, Lviv National University, \\ 1, Universytetska St., 79000, Lviv, Ukraine
}

(Received December 29, 2006)

Present to Professor Ivan Vakarchuk on the occasion of his 60th birthday.

\begin{abstract}
We provide a survey of the results concerning the spaces of measures on compact and ultrametric spaces related to the procedure of dequantization in the sense of Maslov's school.

Key words: ultrametric, measure, idempotent measure, dequantization.
\end{abstract}

PACS number(s): 02.30.Cj, 02.40.Pc

\section{INTRODUCTION}

During the last decades Idempotent Mathematics, i. e. mathematics obtained by replacing the usual arithmetic operations by idempotent ones (an example is the operation $\oplus=\max$, as we have $x \oplus x=x$ for all real numbers $x$ ) has been developed by many authors.

In various papers the idempotent mathematics is regarded as "a result of dequantization of the traditional mathematics over numerical fields as the Planck constant $h$ tends to zero taking imaginary values" [1].

The basic philosophy of the investigations in this direction consists in the following correspondence principle, which is regarded as a counterpart of Bohr's correspondence principle in the quantum theory. The principle asserts that there exists a correspondence between the constructions and results of traditional mathematics and those of idempotent mathematics [1].

An example of such a correspondence is that, if we replace + by max in the definition of metric, the triangle inequality looks as $d(x, y) \leq d(x, z) \oplus d(z, y)$ (strong triangle inequality), i. e. we obtain the notion of ultrametric (another term for "ultrametric" is "non-Archimedean metric").

The notion of (additive) measure in mathematics corresponds to that of idempotent measure. A measure $m$ is idempotent if $m(A \cup B)=\max \{m(A), m(B)\}$. Note that these measures appear also in the fuzzy set theory as "possibility measures" (see [2]).

It is well-known (see, e.g. [3]) that one can regard the compact convex subsets of locally convex spaces as the algebras of the probability measure monad. The fact that the so called max-plus convex sets correspond to the idempotent probability measure monad is remarked in [4]. Recall that a subset $A$ in the Euclidean space $\mathbb{R}^{n}$ is called max-plus convex if, for any $\alpha, \beta \in[-\infty, 0]$ with $\alpha \oplus \beta=0$ and any $x, y \in A$, we have $\alpha \odot x \oplus \beta \odot y \in A$ (here by $\odot$ we denote the addition; note that this definition corresponds, in the idempotent mathematics, to the usual convexity because of the following observation: $-\infty$ is a neutral element for the operation $\oplus$ while 0 is that for the operation $\odot$ ).

A version of max-plus convexity is defined by Briec and
Horvath [5]. For any homeomorphism $\Phi: X \rightarrow \mathbb{R}^{n}$, they define on the topological space $X$ the set operator $\mathrm{Co}^{\Phi}$ by the formula $\operatorname{Co}^{\Phi}(A)=\Phi^{-1}(\operatorname{Co}(\Phi(A)))$, where Co stands for the convex hull in $\mathbb{R}^{n}$. In particular, if $\varphi: \mathbb{R} \rightarrow \mathbb{R}$ is a homeomorphism, one defines $\Phi=\varphi \times \ldots \times \varphi: \mathbb{R}^{n} \rightarrow \mathbb{R}^{n}$. Taking $\varphi_{r}(t)=t^{2 r+1}$, where $r$ is a natural number, one obtains homeomorphisms $\Phi_{r}=\varphi_{r} \times \ldots \times \varphi_{r}$.

The set $A \subset \mathbb{R}_{+}^{n}$ is said to be $\mathbb{B}$-convex if $t x \oplus y \in A$ for any $x, y \in \mathbb{R}^{n}$ and $t \in[0,1]$. The $\mathbb{B}$-convex are precisely the Kuratowski-Painleve limits of the $\Phi_{r}$-convex sets as $r \rightarrow \infty$ (see [5-7]).

We see that the $\mathbb{B}$-convex sets can be obtained from the max-plus convex sets by change of variables $t \mapsto e^{t}$ and replacing addition by multiplication.

The ultrametrization in this paper is a counterpart of the ultrametrization in the set of probability measures with compact support (see [8]).

The paper provides a brief survey of the results obtained in the direction mentioned above. We also formulate some conjectures and open problems.

\section{PRELIMINARIES}

We assume that the reader is familiar with the backgrounds of the category theory (see, e.g. [9]). Given a category $\mathcal{C}$, we denote by $|\mathcal{C}|$ the class of its objects. For brevity, functor stands for covariant functor.

By Comp we denote the category of compact Hausdorff spaces. We usually assume that all maps are continuous. See, e. g. [10] for basic notions of the general theory of functors acting in Comp.

Let $C(X)$ denote the set of continuous functions on a topological space $X$. The set $C(X)$ is a linear space with respect to the operations of pointwise addition and multiplication by scalars and also this set is a lattice with respect to the pointwise operations max and min.

Let $C>0$. A map $f: X \rightarrow Y$ of metric spaces $(X, d)$, $(Y, \varrho)$ is called $C$-Lipschitz if

$$
\varrho(f(x), f(y)) \leq C d(x, y), x, y \in X .
$$

If $C=1$ we say that $f$ is nonexpanding, if $C<1$ we say that $f$ is a contraction. 
If $A$ is a subset of a metric space and $r>0$, by $O_{r}(A)$ we denote the open $r$-neighborhood of $A$.

Recall that a function $d: X \times X \rightarrow \mathbb{R}$ is called a pseudometric on $X$ if
1. $d(x, y) \geq 0, d(x, x)=0$
2. $d(x, y)=d(y, x)$;
3. $d(x, y) \leq d(x, z)+d(z, y)$.

\section{IDEMPOTENT PROBABILITY MEASURES AND MAX-MIN MEASURES}

First, we briefly outline some definitions from [4].

Following the style of idempotent mathematics (see, e.g., $[1,11,12])$ we denote by $\odot: \mathbb{R} \times C(X) \rightarrow C(X)$ the map acting by $(\lambda, \varphi) \mapsto \lambda_{X}+\varphi$, and by $\oplus: C(X) \times$ $C(X) \rightarrow C(X)$ the map acting by $(\varphi, \psi) \mapsto \max \{\varphi, \psi\}$.

For each $c \in \mathbb{R}$ by $c_{X}$ we denote the constant function from $C(X)$ defined by the formula $c_{X}(x)=c$ for each $x \in X$.

Definition III.1 A functional $\mu: C(X) \rightarrow \mathbb{R}$ is called an idempotent probability measure ( $a$ Maslov measure) if

$$
\begin{aligned}
& \text { 1. } \mu\left(c_{X}\right)=c ; \\
& \text { 2. } \mu(c \odot \varphi)=c \odot \mu(\varphi) ; \\
& \text { 3. } \mu(\varphi \oplus \psi)=\mu(\varphi) \oplus \mu(\psi) \text {, }
\end{aligned}
$$

for every $\varphi, \psi \in C(X)$ and every $c \in \mathbb{R}$.

The number $\mu(\varphi)$ is the Maslov integral of $\varphi \in C(X)$ with respect to $\mu$. Another notation for this integral is $\int_{X}^{\oplus} \varphi d \mu$.

Let $I(X)$ denote the set of all idempotent probability measures on $X$. We endow $I(X)$ with the weak* topology. A base of this topology is formed by the sets

$$
\begin{aligned}
& \left\langle\mu ; \varphi_{1}, \ldots, \varphi_{n} ; \varepsilon\right\rangle=\left\{\nu \in I(X)|| \mu\left(\varphi_{i}\right)-\nu\left(\varphi_{i}\right) \mid<\varepsilon,\right. \\
& i=1, \ldots, n\},
\end{aligned}
$$

where $\mu \in I(X), \varphi_{i} \in C(X), i=1, \ldots, n$, and $\varepsilon>0$. Endowed with this topology, the space $I(X)$ is a subspace of the space of the order-preserving weakly additive functionals $O(X)$ introduced by T. Radul $[13,14]$.

The construction $I$ determines a functor on the category Comp. Given the morphism $f: X \rightarrow Y$ in Comp, we define $I(f): I(X) \rightarrow I(Y)$ by the condition: $I(f)(\mu)(\varphi)=$ $\mu(\varphi f), \mu \in I(X), \varphi \in C(Y)$. Note that the functor $I$ is a subfunctor of the functor $O$ of weakly additive orderpreserving functionals $[13,14]$. We also mention another subfunctor of the functor $O$, namely the well-known functor $P$ of probability measures. The space $P(X)$ consists of non-negative normed linear functionals on $C(X)$.

The following is an example of an idempotent probability measure. Let $x_{1}, \ldots, x_{n} \in X$ and $\lambda_{1}, \ldots, \lambda_{n} \in \mathbb{R}$ be numbers such that $\max \left\{\lambda_{1}, \ldots, \lambda_{n}\right\}=0$. Define $\mu: C(X) \rightarrow \mathbb{R}$ as follows:

$$
\mu(\varphi)=\max \left\{\varphi\left(x_{i}\right)+\lambda_{i} \mid i=1, \ldots, n\right\}, \varphi \in C(X) .
$$

As usual, for every $x \in X$, we denote by $\delta_{x}$ (or $\delta(x)$ ) the functional on $C(X)$ defined as follows: $\delta_{x}(\varphi)=\varphi(x)$, $\varphi \in C(X)$ (the Dirac probability measure concentrated at $x)$. Then one can write $\mu=\oplus_{i=1}^{n} \lambda_{i} \odot \delta_{x_{i}}$.

To every $\mu \in I(X)$ there corresponds a function (let us denote it by $m_{\mu}$ ) defined on closed subsets on $X$ :

$$
m_{\mu}(A)=\inf \{\mu(\varphi)|\varphi \in C(X), \varphi| A \equiv 0\}, A \subset X .
$$

This set-valued function satisfies the condition $m_{\mu}(A \cup$ $B)=\max \left\{m_{\mu}(A), m_{\mu}(B)\right\}$, i. e. is a possibility measure (see [2]). Note that the possibility measures take their values in the set $\mathbb{R}_{\max }=[-\infty, 0]$.

Applying the transformation $t \mapsto e^{t}$, we come to the set $J(X)$ of functionals $\mu$ defined on the set $C_{+}(X)$ of positive continuous functions on a compact Hausdorff space $X$ satisfying

1. $\mu\left(c_{X}\right)=c$

2. $\mu(c \varphi)=c \mu(\varphi)$

3. $\mu(\varphi \oplus \psi)=\mu(\varphi) \oplus \mu(\psi)$,

for every $\varphi, \psi \in C(X)$ and every $c \geq 0$. Applying the above transformation to the just defined possibility measures one obtains the measures taking their values in the segment $[0,1]$.

Let $\star$ denote the min operation on the extended real line

$$
\overline{\mathbb{R}}=\mathbb{R} \cup\{-\infty, \infty\}=[-\infty, \infty] .
$$

We endow the set $\overline{\mathbb{R}}$ with the topology generated by its natural order.

Let $X \in|\mathbf{C o m p}|$. We denote by $\Xi(X)$ the set of functionals $\mu: C(X) \rightarrow \mathbb{R}$ satisfying

1. $\mu(\varphi) \oplus \mu(\psi)$;

2. $\mu\left(c_{X}\right)=c$;

3. $\mu(\lambda \star \varphi)=\lambda \star \mu(\varphi)$, for every $\lambda \in \overline{\mathbb{R}}, \lambda>-\infty$;

4. $\mu\left(\varphi+c_{X}\right) \leq \mu(\varphi)+c$

can be topologized by the weak* topology. Similarly to [4] one can prove that $\Xi(X)$ is a compact Hausdorff space if such is $X$. Note that no longer $\Xi(X)$ is a subset of $O(X)$ because of the lack of weak additivity.

If $f: X \rightarrow Y$ is a morphism in Comp, then we define a map $\Xi(f): \Xi(X) \rightarrow \Xi(Y)$ as follows: $\Xi(f)(\mu)(\varphi)=$ $\mu(\varphi f), \mu \in \Xi(X), \varphi \in C(Y)$. If $f: X \rightarrow Y$ is an embedding then such is $\Xi(f)$ and in this case we identify $\Xi(X)$ with the subset $\Xi(f)(\Xi(X))$ of $\Xi(Y)$.

Suppose that $A$ is a closed subset of $X$ and $\mu \in \Xi(X)$. One can define the measure of $A$ as the number

$$
m_{\mu}(A)=\lim _{t \rightarrow \infty} \inf \{\mu(\varphi)|\varphi \in C(X), \varphi| A \equiv t\} .
$$

We obtain again a possibility measure defined on closed subsets in $X$. Unlik the case of the space $I(X)$, the measure $m_{\mu}$ takes its values in the set $\overline{\mathbb{R}}$. 


\section{ZARICHNYI}

The following is an example of an element of $\Xi(X)$. Let $x_{1}, \ldots, x_{n} \in X$ and $\lambda_{1}, \ldots, \lambda_{n} \in \overline{\mathbb{R}}$ be such that $\bigoplus_{i=1}^{n} \lambda_{i}=\infty$. For any $\varphi \in C(X)$, define

$$
\mu(\varphi)=\bigoplus_{i=1}^{n} \lambda_{i} \star \varphi\left(x_{i}\right)=\bigoplus_{i=1}^{n} \lambda_{i} \star \delta_{x_{i}}(\varphi) .
$$

One can easily see that $\mu \in \Xi(X)$.

Proposition III.2 For every $\mu \in \Xi(X)$ with $\operatorname{supp}(\mu)=$ $\left\{x_{1}, \ldots, x_{n}\right\}$ there exist $\lambda_{1}, \ldots, \lambda_{n} \in \overline{\mathbb{R}}$ such that $\mu$ has form (1).

Proof. Without loss of generality, one may assume that $X=\left\{x_{1}, \ldots, x_{n}\right\}$. Define, for every $t>0$ and every $i=1, \ldots, n$, a function $\varphi_{i}^{t} \in C(X)$ by the condition $\varphi_{i}^{t}\left(x_{j}\right)=t$ whenever $i=j$ and $-t$ otherwise. Let $\lambda_{i}=\inf _{t>0} \mu\left(\varphi_{i}^{t}\right)$. We leave to the reader the verification that $\mu$ is then represented by formula (1).

Proposition III.3 Let $X=\left\{x_{1}, \ldots, x_{n}\right\}$ (we suppose that the cardinality of $X$ is $n$, i. e. $x_{i} \neq x_{j}$ whenever $i \neq j)$. Then the space $\Xi(X)$ is homeomorphic to the following "max-min"-simplex

$$
\bar{\Gamma}^{n-1}=\left\{\left(\lambda_{1}, \ldots, \lambda_{n}\right) \in \overline{\mathbb{R}}^{n} \mid \bigoplus_{i=1}^{n} \lambda_{i}=\infty\right\} .
$$

There is a natural coordinatewise homeomorphism of $\Gamma^{n-1}$ onto $\bar{\Gamma}^{n-1}$ induced by the homeomorphism of $\mathbb{R}_{\max }=[-\infty, 0]$ onto $\overline{\mathrm{R}}$ acting by $t \mapsto-\ln (-t)$ on finite points. This homeomorphism naturally extends to a functorial isomorphism of the restrictions of the functors $I$ and $\Xi$ onto the category of finite spaces.

We formulate an open problem whether such an isomorphism can be extended over the whole category Comp. The following argument speaks in favor of the affirmative solution of this problem. Let $m$ be a possibility measure defined on Borel subsets of a compact metric space $X$ and taking its values in $\overline{\mathbb{R}}$. Like in the Lebesgue integral theory, for any simple function $\varphi$ on $X$ (i.e. a function with at most countable image $\varphi(X)$ such that $\varphi^{-1}(y)$ is Borel for every $y \in$ $\varphi(X))$, one can define its max-min-integral $\int_{X} \varphi d m$ as the expression $\bigoplus_{y \in \varphi(X)} y \star m\left(\varphi^{-1}(y)\right)$. Extending the obtained integral over the class of continuous functions by means of the standard procedure one obtains a functional $\mu_{m}: C(X) \rightarrow \mathbb{R}$, which belongs to $\Xi(X)$. In turn, if one defines the integral as $\bigoplus_{y \in \varphi(X)} y \odot m\left(\varphi^{-1}(y)\right)$, one obtains a functional in $I(X)$.

\section{MAX-MIN-MEASURES ON ULTRAMETRIC SPACES}

In [8], the ultrametrics on the set of probability measures on the ultrametric spaces are considered in connections with problems of computer science, in particular, the semantics of program languages. The idempotent measures on ultrametric spaces are introduced in [15].
Now, let $X$ be a metric space. We define the set $\Xi(X)$ as the direct limit

$$
\left.\underset{\lim }{\longrightarrow} F(A), F\left(i_{A B}\right) ; \exp X\right\},
$$

where $\exp X$ is the family of all non-empty compact subsets of $X$ ordered by inclusion and, for every $A, B \in$ $\exp X$ with $A \subset B$ the map $i_{A B}: A \rightarrow B$ is an embedding. We define the support of $\mu \in \Xi(X)$ (written $\operatorname{supp}(\mu)$ ) as the minimal (with respect to inclusion) set $A \in \exp X$ such that $\mu \in \Xi(A)$.

Let $(X, d)$ be an ultrametric space. We are going to endow the set $\Xi(X)$ with an ultrametric. For any $\varepsilon>0$, denote by $\mathcal{F}_{\varepsilon}=\mathcal{F}_{\varepsilon}(X)$ the set of all functions $\varphi \in C(X)$ satisfying the property: for any $y \in \varphi(X)$ the set $\varphi^{-1}(y)$ is the union of balls of radii $\varepsilon$.

Given $\mu, \nu \in I(X)$, we let

$$
\check{d}(\mu, \nu)=\inf \left\{\varepsilon>0 \mid \mu(\varphi)=\nu(\varphi) \text { for all } \varphi \in \mathcal{F}_{\varepsilon}\right\} .
$$

Proposition IV.1 The function $\check{d}$ is ultrametric on $I(X)$.

Proof. Evidently, $\check{d} \geq 0$. Let now $\mu, \nu \in I(X), \mu \neq \nu$. Then there exists $\varphi \in C(X)$ such that $\mu(\varphi) \neq \nu(\varphi)$. Without loss of generality, one may assume that $\mu(\varphi)-$ $\nu(\varphi)=c>0$. Since $\mu, \nu$ are of compact supports, one may assume that there is a compact subset $K \subset X$ such that $\varphi \mid(X \backslash K) \equiv 0$.

One can easily construct $\psi \in \mathcal{F}_{\varepsilon}$, for some $\varepsilon>0$, such that $\psi \leq \varphi \leq \psi+(c / 3)$.

Then

$$
\begin{aligned}
& \mu(\psi) \leq \mu(\varphi) \leq \mu\left(\psi+(c / 3)_{X}\right) \leq \mu(\psi)+(c / 3), \\
& \nu(\psi) \leq \nu(\varphi) \leq \mu\left(\psi+(c / 3)_{X}\right) \leq \mu(\psi)+(c / 3),
\end{aligned}
$$

whence $\mu(\psi) \neq \nu(\psi)$ and therefore $\check{d}(\mu, \nu) \geq \varepsilon>0$.

It is obvious that $\check{d}(\mu, \nu)=\check{d}(\nu, \mu)$, i. e. the function $\check{d}$ is symmetric.

In order to prove the strong triangle inequality, let $\mu, \nu, \tau \in I(X), \check{d}(\mu, \nu)=a, \check{d}(\nu, \tau)=b$. Without loss of generality, we may assume that $a \geq b$. Then, for every $\eta>0$ and every $\varphi \in \mathcal{F}_{a+\eta}$, we have $\mu(\varphi)=\nu(\varphi)$ and $\nu(\varphi)=\tau(\varphi)$. Letting $\eta \rightarrow 0$, we see that $\breve{d}(\mu, \tau) \leq a$.

Endowed with this metric, the space $\Xi_{\omega}(X)$ of the max-min measures of finite supports turns out to be isometric to the space $I_{\omega}(X)$ of the idempotent measures of finite supports via the map sending $\bigoplus_{i=1}^{n} \lambda_{i} \star \delta_{x_{i}}$ to $\bigoplus_{i=1}^{n}-e^{-\lambda_{i}} \oplus \delta_{x_{i}}$. Since one can prove that the set $\Xi_{\omega}(X)$ is dense in $\Xi(X)$ and the corresponding fact holds true for the idempotent measures. This isometry extends to the unique isometry between the spaces $\Xi(X)$ and $I(X)$. Moreover, it turns out that these isometries induce an isomorphism of the functors $\Xi$ and $I$ on the category UMet of the ultrametric spaces and nonexpanding maps. At the first glance, there is no reason to introduce the functor $\Xi$, however, as we will see later, there is a substantial distinction between $\Xi$ and $I$ on the higher level of consideration. 


\section{MONAD STRUCTURE}

\section{A. Category Comp}

Recall that a triple $\mathbb{T}=(F, \eta, \psi)$ is called a monad on a category $\mathcal{C}$ if $F: \mathcal{C} \rightarrow \mathcal{C}$ is a functor and $\eta: 1_{\mathcal{C}} \rightarrow F$, $\psi: F^{2} \rightarrow F$ (hereafter $F^{2}$ denotes the composition $F F$, similarly to $F^{3}$ ) are natural transformations such that the diagrams
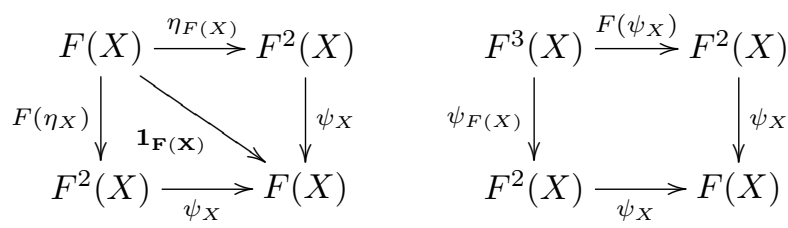

are commutative. If $\mathbb{F}=(F, \eta, \psi)$ and $\mathbb{F}^{\prime}=\left(F^{\prime}, \eta^{\prime}, \psi^{\prime}\right)$ are monads on the same category, $\mathcal{C}$, we say that a natural transformation $\gamma: F \rightarrow F^{\prime}$ is a morphism of the monad $\mathbb{F}$ onto $\mathbb{F}^{\prime}$ if the diagrams
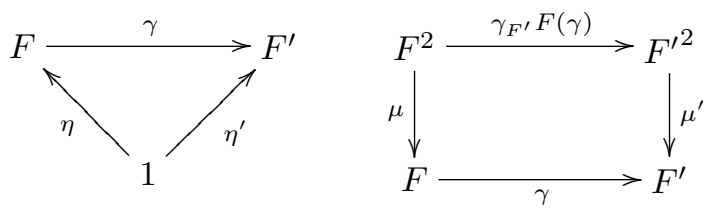

are commutative.

If $\gamma$ consists of embeddings, we say that $\mathbb{F}$ is a $s u b$ monad of $\mathbb{F}^{\prime}$.

The monad structure with the functor $I$ as its functorial part is defined in [4]. It follows from the general theory of functors acting in the category Comp (see [10]) that $\delta: 1_{\text {Comp }} \rightarrow I$ is the unique natural transformation. The natural transformation $\zeta: I^{2} \rightarrow I$ is defined as follows.

Let $X \in|\mathbf{C o m p}|$. Given $\varphi \in C(X)$, define $\bar{\varphi}: I(X) \rightarrow$ $\mathbb{R}$ as follows: $\bar{\varphi}(\mu)=\mu(\varphi), \mu \in I(X)$.

Given $M \in I^{2}(X)$, define the $\operatorname{map} \zeta_{X}(M): C(X) \rightarrow \mathbb{R}$ as follows: $\zeta_{X}(M)(\varphi)=M(\bar{\varphi})$.

The obtained monad $\mathbb{I}=(I, \delta, \zeta)$ turns out to be a submonad of the monad $\mathbb{O}$ of weakly additive orderpreserving functionals introduced by Radul [14]. The probability measure monad $\mathbb{P}$ is also a submonad of $\mathbb{O}$. By change of variables $t \mapsto e^{t}$, one obtains, out of the monad $\mathbb{I}$, the monad $\mathbb{J}$ on the category Comp.

We are going to define a monad structure with $\Xi$ as its functorial part. Let $\varphi \in C(X)$, where $X$ is a compact Hausdorff space. Define $\bar{\varphi}: \Xi(X) \rightarrow \mathbb{R}$ as follows: $\bar{\varphi}(\mu)=\mu(\varphi), \mu \in \Xi(X), \mu \in \Xi(X)$.

Define a map $\bar{\zeta}_{X}$ by the condition: $\bar{\zeta}_{X}(M)(\varphi)=M(\bar{\varphi})$, $\varphi \in C(X)$. If $M=\bigoplus \lambda_{i} \star \mu_{i} \in \Xi^{2}(X)$, where $\mu_{i}=$ $\bigoplus \kappa_{i j} \star \delta_{x_{i j}} \in \Xi(X)$, for every $i$, then

$$
\bar{\zeta}(M)=\bigoplus_{i} \bigoplus_{i j}\left(\lambda_{i} \star \kappa_{i j}\right) \star \delta_{x_{i j}} \in \Xi(X) .
$$

The map $\eta_{X}: X \rightarrow \Xi(X)$ sends $x \in X$ to the Dirac measure $\delta_{x}$ (denoted also $\delta_{X}(x)$ ).

The maps $\bar{\zeta}_{X}$ and $\delta_{X}$ are components of natural transformations $\bar{\zeta}: \Xi^{2} \rightarrow \Xi$ and $\delta: 1_{\mathbf{C o m p}} \rightarrow \Xi$ respectively.

The following result is a counterpart of that obtained for the idempotent probability measure functor [4].

Theorem V.1 The triple $\mathbb{X}=(\Xi, \delta, \bar{\zeta})$ is a monad on the category Comp.

We conjecture that the monads $\mathbb{I}$ and $\mathbb{X}$ are not isomorphic.

Given the $\operatorname{monad} \mathbb{T}=(T, \eta, \mu)$ on the category $\mathcal{C}$, we say that the pair $(X, \xi)$, where $\xi: T(X) \rightarrow X$ is a morphism in $\mathcal{C}$, is a $\mathbb{T}$-algebra if $\xi \eta_{X}=1_{X}$ and $\xi \mu_{X}=\xi T(\xi)$. Given $\mathbb{T}$-algebras $(X, \xi),\left(X^{\prime}, \xi^{\prime}\right)$, we say that a morphism $f: X \rightarrow X^{\prime}$ is a morphism of $\mathbb{T}$-algebras if $f \xi=\xi^{\prime} T(f)$. The $\mathbb{T}$-algebras and their morphisms form a category. The category $\mathbb{P}$-algebras is known to be isomorphic to that of compact convex subsets in locally convex spaces and continuous affine maps. Given such a compact convex set, $X$, one defines the map $\xi: P(X) \rightarrow X$ as the barycenter map defined by the condition: $\varphi(\xi(\mu))=$ $\mu(\varphi)$, for every linear function $\varphi \in C(X)$.

Question V.2 Characterize the category of $\mathbb{X}$-algebras.

We conjecture that the category of $\mathbb{X}$-algebras is that of compact max-min-convex sets in the locally convex lattices. Equivalently, compact max-min-convex sets in the Tychonov product $\mathbb{R}^{\tau}$, where $\tau$ is an arbitrary cardinal number. Here, a set $X \subset \mathbb{R}^{\tau}$ is max-min-convex if, for any $x, y \in X$ and any $t, s \in[-\infty, \infty]$ with $t \oplus s=\infty$, we have $t \star x \oplus s \star y \in X$ (the operations are the coordinatewise maximum and minimum). The morphisms of max-min-convex sets are those induced by linear maps $\mathbb{R}^{\tau} \rightarrow \mathbb{R}^{\tau^{\prime}}$ adjoint to maps $\tau^{\prime} \rightarrow \tau$. The counterpart of the barycenter map, $\xi: \Xi(X) \rightarrow X$ acts as follows:

$$
\xi\left(\bigoplus_{i=1}^{n} \lambda_{i} \star \delta_{x_{i}}\right)=\bigoplus_{i=1}^{n} \lambda_{i} \star x_{i}
$$

(and continuously extends to all the subspace $\Xi(X)$ ). According to the philosophy of monadology, one can regard the spaces of the form $\Xi(X)$ as the free max-min-convex sets.

Summing up, one obtains the following correspondence between the monads and the classes of convex sets in the

\begin{tabular}{|c|c|c|c|}
\hline monad & $\mathbb{P}$ & $\mathbb{I}(\mathbb{J})$ & $\mathbb{X}$ \\
\hline algebras & convex sets & $\begin{array}{c}\text { max-plus convex sets } \\
\text { (BB-convex sets) }\end{array}$ & $\mathrm{n}$ \\
\hline
\end{tabular}
category Comp: 


\section{ZARICHNYI}

That $\mathbb{P}$-algebras are precisely compact convex sets is explicitly shown in [3]; some results of [4] testify to the fact that the max-plus convex sets are the $\mathbb{I}$-algebras.

\section{B. Category of ultrametric spaces}

Now let us turn our attention to the category UMet. That the probability measure functor on the category UMet defined in [8] determines a monad was first remarked by the author. In [15] a counterpart of this monad was constructed with $I$ as its functorial part.

Given an ultrametric space $(X, d)$, we endow the set $\Xi(X)$ with the ultrametric $\breve{d}$. Then the space $\Xi^{2}(X)$ carries the ultrametric $\check{\breve{d}}$.

Proposition V.3 The map $\bar{\zeta}_{X}: \Xi^{2}(X) \rightarrow \Xi(X)$ is nonexpanding.

Now, let $X=\{x, y, z\}$ and

$$
\mu=0 \odot \delta_{x} \oplus(-1) \odot \delta_{y}, \mu^{\prime}=0 \odot \delta_{x} \oplus(-2) \odot \delta_{y},
$$

This allows us to regard the maps $\bar{\zeta}_{X}$ as the components of a natural transformation $\bar{\zeta}: \Xi^{2} \rightarrow \Xi$ in UMet. Together with the natural transformation $\delta: 1_{\text {UMet }} \rightarrow \Xi$ this gives us a monad on the category UMet.

A monad $(I, \delta, \zeta)$ on the category UMet generated by the functor $I$ is defined in [15]. The following example demonstrates that no coordinatewise isomorphism $\gamma$ of the functors $I$ and $\Xi$ induces a monad isomorphism. Namely, let $\alpha:[-\infty, 0] \rightarrow[-\infty, \infty]$ be an orderpreserving continuous homeomorphism (the mentioned homeomorphism $t \mapsto-\ln (-t)$ is an example). Note that, for $Y=\left\{y_{1}, \ldots, y_{k}\right\}$, we define

$$
\gamma_{Y}\left(\bigoplus_{i=1}^{k} c_{i} \odot \delta_{y_{i}}\right)=\bigoplus_{i=1}^{k} \alpha\left(c_{i}\right) \star \delta_{y_{i}} .
$$

Then

$$
\zeta_{X}(M)=c \odot \delta_{x} \oplus(c-1) \odot \delta_{y} \oplus \delta_{z} \neq \zeta_{X}\left(M^{\prime}\right)=c \odot \delta_{x} \oplus(c-2) \oplus \delta_{y} \oplus \delta_{z},
$$

while, under the isomorphism $\gamma$ defined above, we have

$$
\begin{aligned}
\bar{\zeta}_{X}\left(\gamma_{\Xi(X)}\left(I\left(\gamma_{X}(M)\right)\right)\right. & =\alpha(c) \star \delta_{x} \oplus(\alpha(c) \star \alpha(-1)) \star \delta_{y} \oplus \delta_{z} \\
& =\bar{\zeta}_{X}\left(\gamma_{\Xi(X)}\left(I\left(\gamma_{X}(M)\right)\right)=\alpha(c) \star \delta_{x} \oplus(\alpha(c) \star \alpha(-2)) \star \delta_{y} \oplus \delta_{z}\right.
\end{aligned}
$$

whenever $c<-2$.

The underlying spaces of the $\mathbb{X}$-algebras in the category UMet can serve as counterparts of the so-called max-min-convex sets in the category Comp. Similarly, the algebras of the probability measure and possibility measure monads can be interpreted as counterparts of the convex sets, and the max-plus convex sets, respectively.

\section{METRIZATION}

Let $(X, d)$ be a compact metric space.

By $n$-LIP $=n$-LIP $(X, d)$ we denote the set of Lipschitz functions with the Lipschitz constant $\leq n$ from $C(X)$.

Fix $n \in \mathbb{N}$. For every $\mu, \nu \in I(X)$, let

$$
\hat{d}_{n}(\mu, \nu)=\sup \{|\mu(\varphi)-\nu(\varphi)| \mid \varphi \in n-\operatorname{LIP}\} .
$$

The following theorem is proved in [4].

Theorem VI.1 The function $\hat{d}_{n}$ is a continuous pseudometric on $I(X)$.
One can similarly prove that the function $\check{d}_{n}: \Xi(X) \times$ $\Xi(X) \rightarrow \mathbb{R}$ defined by the formula

$$
\check{d}_{n}(\mu, \nu)=\sup \{|\mu(\varphi)-\nu(\varphi)| \mid \varphi \in n-\operatorname{LIP}\}
$$

is a continuous pseudometric on $\Xi(X)$.

Proposition VI.2 The map $\delta=\delta_{X}, x \mapsto \delta_{x}:(X, d) \rightarrow$ $\left(I(X), \tilde{d}_{n}\right)$, is an isometric embedding for every $n \in \mathbb{N}$.

A similar fact takes place also for the pseudometric $\check{d}_{n}$ on $\Xi(X)$.

Note that the above construction of $\hat{d}$ can be applied not only to metrics but also to continuous pseudometrics. Proceeding in this way we obtain the iterations $\left(I(X), \tilde{d}_{n}\right),\left(I^{2}(X), \tilde{\tilde{d}}_{n m}=\left(\tilde{d}_{n}\right)_{m}\right), \ldots$

Proposition VI.3 For a metric space $(X, d)$, the map $\zeta_{X}:\left(I^{2}(X), \tilde{\tilde{d}}_{n n}\right) \rightarrow\left(I(X), \tilde{d}_{n}\right)$ is nonexpanding.

The latter proposition has its counterpart also for the pseudometrics $\breve{d}_{n}$ on the spaces $\Xi(X)$.

One way to attack the problem whether the functors $I$ and $\Xi$ are isomorphic consists in tackling the obtained 
pseudometric spaces $\left(I(X), \hat{d}_{n}\right)$ and $\left(\Xi(X), \check{d}_{n}\right)$. Namely, the problem reduces to that whether the map

$$
\bigoplus_{i=1}^{n} \lambda_{i} \star \delta_{x_{i}} \mapsto \bigoplus_{i=1}^{n}-e^{-\lambda_{i}} \oplus \delta_{x_{i}}
$$

is uniformly continuous (and so is its inverse) when the corresponding measure spaces are endowed with the metrics defined above.

\section{ITERATED FUNCTION SYSTEMS}

Let $(X, d)$ be a metric space. An iterated function system in $X$ is a finite sequence $f_{1}, \ldots, f_{n}: X \rightarrow X$ of continuous maps. Fix $\mu_{0} \in \Xi(\{1, \ldots, n\})$. Then $\mu_{0}=\bigoplus_{i=1}^{n} \lambda_{i} \star$ $\delta_{i}$. Any such system determines a map $\Phi: \Xi(X) \rightarrow \Xi(X)$ as follows:

$$
\Phi(\mu)=\bigoplus_{i=1}^{n} \lambda_{i} \star \Xi\left(f_{i}\right)(\mu) .
$$

Dissimilar to the case of the probability measure functor and the Kantorovich metric on the spaces $P(X)$, it does not seem to be true that the maps $\Xi\left(f_{i}\right)$ are contractions (with respect to the family of pseudometrics defined in the previous section) if such are the maps $f_{i}$. This does not allow us to apply, for the map $\Phi$, the Banach contraction principle in order to prove the existence of a unique fixed point of $\Phi$.

One can prove that, for a compact metric $X$, the space $\Xi(X)$ carries a natural generalized convexity structure making it an absolute extensor (AE). Therefore, the map $\Phi$ has a (not necessarily unique) fixed point. We leave as an open problem that of searching conditions that should be imposed onto the iterated function system making these fixed points counterparts of the probabilistic fractals, i. e. possibility fractals.

\section{REMARKS AND OPEN QUESTIONS}

\section{A. Prokhorov type metrics}

Recall that the Prokhorov metric on the set of probability measures on a metric space $(X, d)$ is defined by the formula

$$
d_{P}\left(m, m^{\prime}\right)=\inf \left\{\varepsilon>0 \mid m(F) \leq m^{\prime}\left(O_{\varepsilon}(F)\right)+\varepsilon, m^{\prime}(F) \leq m\left(O_{\varepsilon}(F)\right)+\varepsilon, F \text { closed }\right\} .
$$

It is not clear whether the Prokhorov metric has its counterpart for the ultrametric spaces. The counterpart of the Prokhorov metric can also be defined for the spaces $I(X)$ as well as $\Xi(X)$.

\section{B. Economic applications}

Given a game represented by two payoff functions $u_{i}: X_{1} \times X_{2} \rightarrow \mathbb{R}, i=1,2$, one can define a game in mixed strategies $\bar{u}_{i}: P\left(X_{1}\right) \times P\left(X_{2}\right) \rightarrow \mathbb{R}$, where

$$
\bar{u}_{i}(\mu, \nu)=\int_{X_{1} \times X_{2}} u_{i} d(\mu \otimes \nu), \quad i=1,2 .
$$

Here $\otimes$ stands for the product of probability measures. It is known that the monad structure allows us to define the products of the idempotent probability measures as well as of the max-min-measures. Therefore, one can consider the games in strategies taking their values in the spaces $I(X)$ and $\Xi(X)$. One of the main problems in this direction is to find suitable economic interpretations of these games.
[1] G. L. Litvinov, V. P. Maslov, IHES/M/95/33, Bures-surYvette (1995).

[2] L. A. Zadeh, Fuzzy Sets and Systems 1, 3 (1978).

[3] T. Świrszcz, Bull. Acad. Polon. Sci. Ser. Sci. Math. Astronom. Phys. 22, 39 (1974).

[4] M. Zarichnyi, preprint math/0608754v1(2006).

[5] W. Briec, C. Horvath, Optimization 53, 103 (2004).
[6] W. Briec, C. D. Horvath, A. Rubinov, Pac. J. Optim. 1, 13 (2005)

[7] G. Adilov, A. M. Rubinov, Numer. Funct. Anal. Optim. 27, 237 (2006).

[8] E. P. de Vink, J. J. M. M. Rutten, Theoretical Computer Science 221(1/2), 271 (1999).

[9] M. Barr, Ch. Wells, Toposes, triples and theories (Berlin, 


\title{
M. ZARICHNYI
}

Springer-Verlag, 1985).

[10] E. V. Shchepin, Usp. Mat. Nauk 31, 3 (1981).

[11] G. L. Litvinov, V. P. Maslov, G. B. Shpiz, in: Asymptotic combinatorics with application to mathematical physics (St. Petersburg, 2001), p. 267; in: NATO Sci. Ser. II Math. Phys. Chem. (Kluwer Acad. Publ., Dordrecht, 2002), p. 77 .
[12] G. L. Litvinov, V. P. Maslov, G. B. Shpiz, Math. Notes 69, 696 (2001).

[13] T. Radul, Bull. Polish Acad. Sci. Math. 47, 53 (1999).

[14] T. Radul, Comment. Math. Univ. Carolin. 39, 609 (1998).

[15] O. Hubal, M. Zarichnyi, J. Math. Anal. Appl. (in print).

\section{ПРОСТОРИ МІР, ПОВ'ЯЗАНІ З ДЕКВАНТУВАННЯМ}

\author{
М. Зарічний \\ Механіко-математичний факультет Львівсъкого національного університету імені Івана Франка \\ вул. Університетсъка, 1, Лъвів, 79000, Украӥна
}

Запропоновано огляд результатів, що стосуються просторів мір на компактах й ультраметричних просторів, які пов'язані з процедурою деквантування в сенсі школи Маслова. 